\title{
Perceptual Evaluation of Motion Editing for Realistic Throwing Animations
}

\author{
MICHELE VICOVARO, Department of General Psychology, University of Padua \\ LUDOVIC HOYET, Graphics, Vision and Visualisation Group, Trinity College Dublin \\ LUIGI BURIGANA, Department of General Psychology, University of Padua \\ CAROL O'SULLIVAN, Disney Research, Los Angeles and Trinity College Dublin
}

\begin{abstract}
Animation budget constraints during the development of a game often call for the use of a limited set of generic motions. Editing operations are thus generally required to animate virtual characters with a sufficient level of variety. Evaluating the perceptual plausibility of edited animations can therefore contribute greatly towards producing visually plausible animations. In this article, we study observers' sensitivity to manipulations of overarm and underarm biological throwing animations. In the first experiment, we modified the release velocity of the ball while leaving the motion of the virtual thrower and the angle of release of the ball unchanged. In the second experiment, we evaluated the possibility of further modifying throwing animations by simultaneously editing the motion of the thrower and the release velocity of the ball, using dynamic time warping. In both experiments, we found that participants perceived shortened underarm throws to be particularly unnatural. We also found that modifying the thrower's motion in addition to modifying the release velocity of the ball does not significantly improve the perceptual plausibility of edited throwing animations. In the third experiment, we modified the angle of release of the ball while leaving the magnitude of release velocity and the motion of the thrower unchanged, and found that this editing operation is efficient for improving the perceptual plausibility of shortened underarm throws. Finally, in Experiment 4, we replaced the virtual human thrower with a mechanical throwing device (a ramp) and found the opposite pattern of sensitivity to modifications of the release velocity, indicating that biological and physical throws are subject to different perceptual rules. Our results provide valuable guidelines for developers of games and virtual reality applications by specifying thresholds for the perceptual plausibility of throwing manipulations while also providing several interesting insights for researchers in visual perception of biological motion.
\end{abstract}

Categories and Subject Descriptors: I.3.7 [Computer Graphics]: Three Dimensional Graphics and Realism—Animation General Terms: Experimentation, Human Factors

Additional Key Words and Phrases: Perception, graphics, motion capture, physics, motion editing

ACM Reference Format:

Michele Vicovaro, Ludovic Hoyet, Luigi Burigana, and Carol O’Sullivan. 2014. Perceptual evaluation of motion editing for realistic throwing animations. ACM Trans. Appl. Percept. 11, 2, Article 10 (May 2014), 23 pages.

DOI: http://dx.doi.org/10.1145/2617916

This work is supported by the SFI Captavatar project.

Authors' addresses: M. Vicovaro, Dipartimento di Psicologia Generale, Università degli Studi di Padova, via Venezia 8, 35131 Padova, Italy; email: vicovaro85@gmail.com; L. Hoyet, Trinity College Dublin, Lloyd Institute, Dublin 2, Ireland; email: hoyetl@tcd.ie; L. Burigana, Dipartimento di Psicologia Generale, Università degli Studi di Padova, via Venezia 8, 35131 Padova, Italy; email: luigi.burigana@unipd.it; C. O’Sullivan, Disney Research, Los Angeles, 5621 Circle Seven Drive, Glendale, CA 91201; email: carol.a.osullivan@disney.com.

Permission to make digital or hard copies of part or all of this work for personal or classroom use is granted without fee provided that copies are not made or distributed for profit or commercial advantage and that copies show this notice on the first page or initial screen of a display along with the full citation. Copyrights for components of this work owned by others than ACM must be honored. Abstracting with credit is permitted. To copy otherwise, to republish, to post on servers, to redistribute to lists, or to use any component of this work in other works requires prior specific permission and/or a fee. Permissions may be requested from Publications Dept., ACM, Inc., 2 Penn Plaza, Suite 701, New York, NY 10121-0701 USA, fax +1 (212) 869-0481, or permissions@acm.org.

(c) 2014 ACM 1544-3558/2014/05-ART10 $\$ 15.00$

DOI: http://dx.doi.org/10.1145/2617916 


\section{INTRODUCTION}

The study of visual perception of physical distortions has a long history, originating with studies on naïve physics [Bozzi 1959; McCloskey et al. 1980]. As physical events are an essential part of virtual reality (VR) applications and computer games, researchers in the field of computer graphics have recently started to investigate observers' sensitivity to physical distortions in realistic mechanical simulations involving simple objects [O'Sullivan et al. 2003; Reitsma and O'Sullivan 2009; Yeh et al. 2009] or virtual human characters [Reitsma et al. 2008; Hoyet et al. 2012]. Among other applications, these studies are necessary to provide guidelines for motion editing operations. As animators may need to introduce physical distortions in animations to achieve particular goals, measuring observers' sensitivity to anomalies can contribute greatly towards producing visually plausible animations [Barzel et al. 1996]. Because the visual perception of interactions between simple inanimate objects has typically been studied independently of the perception of interacting humans, little is known about the perception of interactions between virtual human characters and simple inanimate objects.

In this article, we explore how observers perceive modifications of virtual throwing animations introduced by different editing methods, thereby extending our previous work [Vicovaro et al. 2012]. As animation budget constraints during the development of a game often call for the use of a limited set of generic motions, manipulations of the throwing distance of a ball could then be helpful, particularly in sports games where a character has to throw a ball at different distances on the pitch (e.g., EA Sports Madden NFL ${ }^{\mathrm{TM}}$, Sony CE MLB 12: The Show ${ }^{\mathrm{TM}}$, $2 \mathrm{~K}$ Sports NBA $2 \mathrm{~K} 12^{\mathrm{TM}}$ ). Our goal is to investigate, through perceptual experiments, the best ways to modify throwing animations while preserving their perceptual plausibility. Thereby, we also expect to obtain a better understanding of how such actions are perceived. Using two types of throwing motions (overarm and underarm, Figure 1, top) captured from a real actor and displayed on a virtual character, we first modified the release velocity of the ball while leaving the motion of the virtual thrower and the angle of release of the ball unchanged (Experiment 1). In Experiment 2, we evaluated the possibility of further modifying these throwing animations by simultaneously editing the motion of the thrower and the release velocity of the ball, using dynamic time warping (DTW). In Experiment 3, we investigated observers' sensitivity to a third kind of editing operation - that is, modifying the angle of release of the ball while leaving the magnitude of the release velocity and the motion of the thrower unchanged. Finally, in Experiment 4, we replaced the virtual human character with a virtual ramp (Figure 1, bottom) to evaluate whether the pattern of observers' sensitivity to distortions is specific to human throws or if it also generalizes to throws performed by a mechanical device.

Overall, we found that observers tolerate large increases in the release velocity of the ball (Experiment 1). This suggests that animators can use this simple editing operation to lengthen the throwing distance of overarm and underarm throws to a large extent. However, observers are less tolerant to decreases in the release velocity, particularly for underarm throws. In addition, modifying the preparatory motion using DTW to match the modifications of the release velocity (Experiment 2) does not improve the acceptability of edited throwing animations any more than modifying only the release velocity of the ball. In Experiment 3, we found that modifying only the angle of release of the ball is effective for improving the perceptual plausibility of shortened underarm throws. Finally, in Experiment 4, we found that replacing the virtual human character with the virtual ramp reverses the pattern of sensitivity to distortions: observers become more tolerant to decreases in the release velocity of the ball than to increases. Our results thus provide valuable guidelines for developers of games and VR applications by specifying thresholds for the perceptual plausibility of simple manipu-

lations of throwing actions. Moreover, the results provide several interesting insights for researchers in visual perception of biological motion, which will be discussed in Section 9 . 

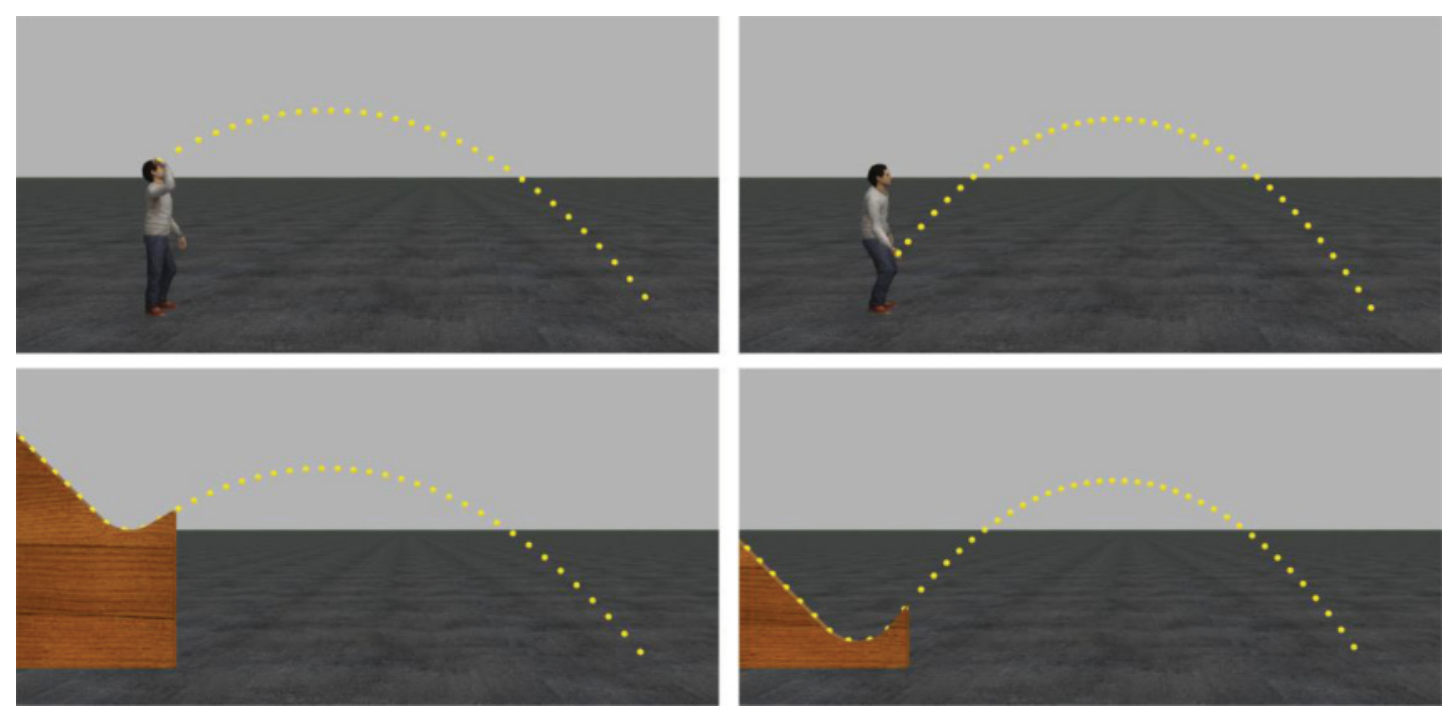

Fig. 1. Examples of biological overarm (top left) and underarm (top right) throws. Corresponding ramp models (bottom) used in the Mechanical Device experiment.

\section{BACKGROUND}

Researchers in naïve physics have shown that students with high school physics instruction have significant misconceptions about elementary mechanics [Clement 1982]. For instance, in paper-andpencil tests, they consistently predicted that a ball rolling inside a curved tube would follow a curved trajectory even when it exits from the tube, whereas it will instead move in a straight line [McCloskey et al. 1980]. Conversely, whereas participants failed in these abstract situations, they performed well when presented with virtual simulations of physical events [Kaiser et al. 1992].

In recent years, researchers in computer graphics have become interested in evaluating by how much a physically correct animation can be modified and still look perceptually plausible [Barzel et al. 1996]. Understanding whether observers are sensitive to physical distortions in mechanical events is important to develop plausible simulations while saving time on details that observers cannot perceive. For instance, the behaviour of a single inanimate object [Kaiser et al. 1992; Nusseck et al. 2007] and sensitivity to errors in 3D rigid body collisions between simple objects [O'Sullivan et al. 2003; Reitsma and O'Sullivan 2009] have been investigated. Motion capture has also been used to evaluate observers' sensitivity to errors in the motion of virtual human characters [Chaminade et al. 2007] or in physical interactions between virtual characters [Hoyet et al. 2012]. Similarly, Majkowska and Faloutsos [2007] studied user sensitivity to errors in aerial human motions and found that participants were not sensitive to even significant changes in angular momentum during ballistic motion. To compare human and inanimate motions, Reitsma et al. [2008] evaluated observers' ability to detect errors in the ballistic motion of a virtual human character and of a virtual ball, and found greater sensitivity to variations in the coefficient of gravity when the actor was a human character.

Throwing actions, as discussed in this article, are mechanical interactions between human characters and inanimate objects. A small number of studies have been concerned with the visual perception of such actions. For instance, Runeson and Frykholm [1983] displayed point-light characters throwing an unseen $2.5 \mathrm{~kg}$ sandbag at different distances and found that participants accurately estimated the length of the throw. Munzert et al. [2010] found that observers finely discriminated between the 
travelled distances of a $600 \mathrm{~g}$ ball when only point-light displays of the arm of the thrower were shown. These results suggest that observers can use the kinematic information provided by the motion of the thrower to make accurate predictions of the landing position of the thrown projectile. Indeed, when the projectile is relatively heavy, the force exerted by the thrower can be perceptually inferred from the kinematics of its biological motion. However, nonkinematic cues, such as the direction of the thrower's gaze when throwing light darts towards a target, have also been found to influence observers' ability to predict the final position of the projectile [Knoblich and Flach 2001]. Hecht and Bertamini [2000] presented 2D stick characters and mannequin-like 3D characters throwing small projectiles, and they found that observers were relatively insensitive to added acceleration during the first phase of the ballistic motion of the projectile. On the whole, these findings suggest that the mass of the projectile is a critical factor in determining observers' sensitivity to inconsistencies between the thrower's motion and the motion of the projectile, with sensitivity tending to increase with the mass of the projectile. Moreover, observers' sensitivity to anomalies in biological motion also depends on the visual depiction of the human character [Hodgins et al. 1998] and usually increases with its anthropomorphism [Chaminade et al. 2007]. This suggests that anomalies in throwing animations might be more easily detectable on realistic virtual human characters than on mannequin-like or point-light characters.

Concurrent with these perceptual studies, several researchers also focused on editing human motion to satisfy new constraints and distortions. Early works focused on editing the kinematics of the motion, such as retargeting motions to new characters [Gleicher 1998], introducing additional kinematic constraints [Gleicher 1997], changing the trajectory of a motion [Gleicher 2001], or blending between several animations [Guo and Robergé 1996; Park et al. 2004]. More recently, several researchers have started to explore how to edit the dynamics of the motion using dynamic filters [Pollard and Reitsma 2001; Shin et al. 2003; Yamane and Nakamura 2003; Tak and Ko 2005] or using data-driven approaches [Komura et al. 2005; Zordan et al. 2005]. Whereas many approaches edit the motion using spacetime optimization problems [Liu et al. 2006], different real-time solutions also exist [Shin and Oh 2006]. Moreover, most of the editing approaches require the timing of the animation to be modified, either speeding it up or slowing it down, using a process known as time warping. It is also possible to handle nonuniform compressions or dilatations of parts of a motion by varying the speed modification over time using DTW, which is commonly used in computer animation to synchronize motion sequences with different durations [Bruderlin and Williams 1995; Hsu et al. 2007].

\section{OVERVIEW}

The study presented in this article is an extension of a previous work where we investigated observers' sensitivity to distortions in virtual throwing animations [Vicovaro et al. 2012]. In that study, participants were presented with animations of a realistic virtual human character throwing a tennis ball with either an overarm or underarm motion. In the first experiment, we used DTW to edit the biological throwing motion and modified the release velocity of the ball accordingly, thereby preserving the consistency between the motion of the thrower and that of the ball. In the second experiment, we separately modified the horizontal and vertical components of the release velocity of the ball while leaving the motion of the thrower unchanged, which created a mismatch between the thrower's motion and the motion of the ball. In both experiments, we measured participants' sensitivity to manipulations and consistently found that their sensitivity depended on the interaction between the sign of the manipulation (speed decrease vs. speed increase) and the type of throw (overarm vs. underarm). A nonstatistical comparison between the results of the two experiments revealed that animations edited with DTW were perceptually more plausible than those edited by modifying only one component of the speed of the ball at a time. We suggested that preserving the consistency between the thrower's motion and the motion of the ball improves the perceptual plausibility of edited throwing animations. 
However, the second experiment differed from the first one not only because of the mismatch between the thrower's motion and the motion of the ball but also because modifying separately the horizontal and vertical components of the release velocity introduced variations in the angle of release of the ball. We thus cannot exclude the possibility that the variations of the angle of release, rather than the mismatch, produced the greater sensitivity for this kind of editing operation. Moreover, the observed interaction between speed modification (decrease vs. increase) and type of throw (overarm vs. underarm) requires further validation and explanations. Therefore, the study presented in this article extends on this previous work to further investigate observers' sensitivity to distortions in virtual throwing animations through four separate experiments while addressing some of the questions left open by the previous study.

In Experiment 1, we present the participants with a virtual human character throwing a tennis ball and modify the release velocity of the ball while leaving the thrower's motion and the angle of release of the ball unchanged. This editing operation creates a mismatch between the thrower's motion and the motion of the ball. In Experiment 2, we evaluate the possibility of increasing the perceptual plausibility of edited animations by eliminating this mismatch. We therefore present the participants with the same animations as in Experiment 1 but modify the velocity of the thrower's motion using DTW and then modify the release velocity of the ball accordingly. Experiment 2 is the same as the first experiment in Vicovaro et al. [2012], but it is presented here with a larger number of participants. In Experiment 3, we test the perceptual plausibility of a different kind of editing operation-that is, modifying the angle of release of the ball while leaving the thrower's motion and the magnitude of release velocity unchanged. As in Reitsma et al. [2008], we also want to directly compare observers' sensitivity to modifications in "biological" and "mechanical" throws. Thus, in Experiment 4, we replace the virtual human thrower with a virtual ramp: after the descent along the ramp, the tennis ball is released with physical parameters (position and velocity) matching those of the throwing animations in Experiment 1 (Figure 1). We manipulate the release velocity of the ball while leaving its angle of release and the motion along the descent unchanged. In each experiment, we used the same psychophysical method (i.e., randomly interleaved staircases) and measured observers' sensitivity to editing operations by fitting psychometric curves to individual data. Participants were always presented with two kinds of throws (overarm and underarm) and two kinds of manipulations (increases and decreases) of the relevant variable - that is, magnitude of the release velocity in Experiments 1, 2, and 4 and angle of release in Experiment 3.

\section{SETUP}

\subsection{Motion Capture}

We recorded the full-body movements of a right-handed male actor (thrower hereafter). The thrower was nonprofessional and did not have any specific experience with sports involving throwing a ball. All throws were performed with the right arm using a standard tennis ball as the projectile (diameter $\approx$ $7 \mathrm{~cm}$, mass $\approx 60 \mathrm{~g}$ ). Another person served as receiver but was not recorded. The receiver stood in front of the thrower at a distance of $5 \mathrm{~m}$. The thrower was instructed to look in front of him during the throw and to avoid lateral movement of the ball. The trajectory of the ball was thus mainly displaced in two dimensions with respect to the thrower: forwards and upwards.

As we wished to determine whether observers' sensitivity to errors in throwing animations depended on the way in which the throw is performed, the thrower was instructed to throw the ball to the receiver in two alternative ways: either with an overarm motion or with an underarm motion (Figure 1, and see the supplementary video for detailed examples). We registered three takes for each kind of throw, whereas other takes were discarded due to excessive lateral movement of the ball. For the experiments 
Table I. Horizontal $v_{H O}$ and Vertical $v_{V O}$ Release Velocities for the Three Takes of the Two Types of Captured Human Throws

Velocities for the takes selected for the experiments are presented in bold.

\begin{tabular}{|c|c|c|c|}
\cline { 3 - 4 } \multicolumn{2}{c|}{} & $v_{H 0}(\mathrm{~m} / \mathrm{s})$ & $v_{V 0}(\mathrm{~m} / \mathrm{s})$ \\
\hline \multirow{3}{*}{ Overarm } & Take1 & 5.44 & 3.55 \\
\cline { 2 - 4 } & Take2 & $\mathbf{5 . 3 5}$ & $\mathbf{3 . 3 9}$ \\
\cline { 2 - 4 } & Take3 & 5.76 & 3.23 \\
\hline
\end{tabular}

\begin{tabular}{|c|c|c|c|}
\cline { 3 - 4 } \multicolumn{2}{c|}{} & $v_{H 0}(\mathrm{~m} / \mathrm{s})$ & $v_{V 0}(\mathrm{~m} / \mathrm{s})$ \\
\hline \multirow{3}{*}{ Underarm } & Take1 & 4.44 & 5.40 \\
\cline { 2 - 4 } & Take2 & 4.18 & 5.17 \\
\cline { 2 - 4 } & Take3 & $\mathbf{4 . 4 2}$ & $\mathbf{5 . 4 7}$ \\
\hline
\end{tabular}

presented in this article, we finally selected a single take for each captured throw. This choice was driven by our experimental designs - that is, to avoid the confounding factor of having multiple takes per throw in the staircase procedure-and justified by qualitative observations of the three takes, which revealed high similarities between physical parameters (Table I). Therefore, for each kind of throw, we selected the take with the release velocity closest to the average velocity of the three captured takes. We only refer to these selected takes in the following of the article.

Motion capture was conducted using a 19-camera Vicon optical system, and 55 markers were placed on the body of the thrower. To simultaneously capture the motion of the hand and of the fingers, we placed six extra markers on each hand: two markers on the thumb and one marker on the fingertip of each finger, as in Hoyet et al. [2012]. We also placed four markers on the tennis ball so that they formed the vertices of a tetrahedron and thus did not have any appreciable influence on the trajectory of the ball. This allowed us to estimate the position of the centre of the ball during the entire captured motion. The body and the ball motions were captured at $120 \mathrm{~Hz}$.

\subsection{Physics of Projectiles}

The motion of a thrown object can be divided into two phases: the motion before it is released (preparatory motion) and the motion after the release (ballistic motion). When thrown in the air, an object that is subject only to the force of gravity and to air resistance is called a projectile. If we neglect air resistance, a projectile always follows a parabolic trajectory, completely determined by its horizontal and vertical velocities at the time of release $\left(v_{H O}\right.$ and $v_{V O}$, respectively). More precisely, the parabolic trajectory of a projectile is characterized by the following two equations:

$$
v_{H}(t)=v_{H 0} \quad \text { and } \quad v_{V}(t)=g t+v_{V 0},
$$

where $v_{H}(t)$ and $v_{V}(t)$ are horizontal and vertical velocities, $g$ is the coefficient of gravity, and $t$ is time. The parabolic trajectory of a projectile is then obtained by integrating these equations over time and depends on the release position of the projectile.

Although Equation 1 refers to the ballistic phase of the motion, the release velocities $v_{H 0}$ and $v_{V 0}$ are determined by the motion of the object during preparatory motion. In the case of a throw performed by a human, preparatory motion refers to all movements of the human's body that influence the release velocities of the projectile, such as the motion of the arm and shoulder.

To manipulate the velocity of the projectile, we first needed to determine the time of release $t_{0}$ to discriminate between the preparatory and the ballistic phases. To automatically compute $t_{0}$, we selected the set of eight markers on the right hand of the thrower $\left(s_{t}\right)$ and another set $\left(s_{p}\right)$ consisting of the four markers on the projectile. We then computed the sum of the squared distance between every combination of pairs of markers $\left(m_{t}, m_{p}\right)$ :

$$
d=\sum_{m_{t} \in s_{t}} \sum_{m_{p} \in s_{p}} \operatorname{dist}\left(m_{t}, m_{p}\right)^{2},
$$


where $\operatorname{dist}\left(m_{t}, m_{p}\right)$ is the Euclidean distance between markers $m_{t}$ and $m_{p}$. Then, $t_{0}$ corresponds to the time when the derivative of $d$ exceeds a manually selected threshold-that is, when the variation of the distance between the ball markers and the hand markers differed from capture noise.

We then used the captured trajectory of the projectile during the ballistic phase to automatically compute the release velocities that best fit the whole ballistic motion. Table I shows the release velocities of the ball for the three captured takes of overarm or underarm throws. It also highlights the takes selected for the experiments, selected to be those with the release velocity closest to the average velocity of the three captured takes. Note that in overarm throws the horizontal component exceeds the vertical component, whereas the opposite is true for underarm throws.

\section{EXPERIMENT 1: BALLISTIC MOTION EDITING}

In this experiment, we are interested in studying observers' sensitivity to manipulations of the ballistic motion in biological human throwing animations. We modified the animations using a simple editing operation by modifying only the release velocity of the ball while leaving the preparatory motion of the virtual character and the angle of release of the ball unmodified. This editing operation introduces a physical mismatch between preparatory and ballistic motions, because the latter is modified while the former remains unchanged. We want to compare the results of this experiment with those of the second experiment in our previous study [Vicovaro et al. 2012], where we modified not only the release velocity of the ball but also its angle of release.

The results show that observers' sensitivity to speed modifications depends on the fine features (overarm vs. underarm) of the action being performed (a throw), with observers being particularly sensitive to speed decreases in the underarm throw and quite insensitive to speed increases in both types of throw. The comparison with our previous study reveals that modifying only the release velocity of the ball, rather than modifying both the release velocity and the angle of release, increases the magnitude at which speed modifications are tolerated.

\subsection{Methods and Stimuli}

To display the biological human motions, we selected a virtual character who roughly matched the morphology of our actor (Figure 1, top). The captured body motion was then mapped onto a skeleton, where joint angles were computed and used to drive the virtual character. The ballistic motion was modified by manipulating the original release velocity of the ball, whereas the preparatory motion was left unchanged. Both the horizontal and the vertical components of the original release velocity were modified by the same percentage (differently from our previous study [Vicovaro et al. 2012]), thereby modifying the magnitude of the release velocity without changing the angle of release of the ball. The modified parabolic trajectory of the projectile was then recomputed according to Equation 1 using the modified release velocities.

5.1.1 Psychophysical Method. To accurately determine perceptual thresholds for modifications of the release velocity, we used a randomly interleaved staircase design [Cornsweet 1962], with fixed up and down step sizes. The staircase (or up-down) method is an efficient psychophysical technique for identifying thresholds, because it ensures that most of the trials are presented near the threshold for that particular observer. The ascending staircase starts with the unmodified throw and increases the magnitude of speed modification until the observer perceives the stimulus as incorrect. The magnitude of speed modification is then decreased until the observer perceives the stimulus as correct, then increased until it results in another incorrect response. This up-down process is repeated until a prespecified number of reversals is obtained. As suggested by Garcia-Pérez [2001], we used a down/up step ratio of 0.871 and set the stopping condition to be eight reversals. This ascending staircase is 
complemented by a descending staircase, which starts at a clearly suprathreshold level (i.e., the stimulus is glaringly incorrect) and decreases until a correct response is given. It then reverses course and follows the same reversal process as described previously. To avoid observers anticipating the next stimulus (and hence biasing their response), trials from several staircases are interleaved; the trials thus appear random to the observer.

This psychophysical method elicits a sufficient number of binary responses close to the absolute threshold level to allow a psychometric curve to be fitted to the data. The psychometric curve is a mathematical model representing how the observer's response to the stimuli varies depending on the variation of these stimuli. This procedure allows us to calculate the acceptance threshold-that is, the magnitude of speed modification of the original throw at which the animation is perceived as correct $50 \%$ of time. We also calculate the just noticeable difference (JND), which is the magnitude of modification of the acceptance threshold that would increase or decrease the probability of a correct response by $25 \%$. Here, we refer to JND as a measure of the sharpness of participants' discrimination between correct and incorrect animations: the smaller the JND, the clearer the distinction between correct and incorrect animations.

5.1.2 Stimuli. Based on a pilot study, the Magnitude of modifications of the release velocity varied between $0 \%$ and $90 \%$ of the original release velocity. The Sign of the manipulation of the speed was either a decrease or an increase. There were two kinds of Throws (overarm or underarm).

Therefore, the experiment consisted of eight staircases: 2 Staircase direction (ascending, descending) $\times 2$ Throw (overarm, underarm) $\times 2$ Sign (decrease, increase). To avoid any anticipatory effect, the eight staircases were randomly interleaved. For each participant, in each of the four experimental conditions ( 2 Throw $\times 2$ Sign), we merged the data of the ascending and descending staircases to compute the acceptance threshold and the JND (see Section 5.2).

We selected a camera viewpoint to the right of the thrower (Figure 1), where the fixed position of the camera was chosen to maximize the amount of preparatory and ballistic motion information available to participants. The ball was displayed with a bright-yellow photographic tennis ball texture (similar to the real captured tennis ball), and the ground was displayed with a dark grey asphalt-like textured plane. The background was light grey, and shadows were not rendered. These settings were chosen to enhance the contrast between the ball and the rest of the virtual environment, thus making the visual tracking of the ball easier. Because we wanted the participants to focus on the trajectory of the ball during its flight phase and not on the reaction with the environment once the ball landed, the ball disappeared before making contact with the ground. For some modified throws, the ball went outside of the border of the screen. We did not simulate air resistance because this would have a negligible perceptual effect on the trajectory of the ball. As some of the animations contained a highly dynamic motion of the ball, all of the stimuli were displayed at a resolution of $1,600 \times 1,200$ and at $85 \mathrm{~Hz}$ on a 19-inch CRT screen, thereby preventing unrealistic motion blur present on standard LCD monitors from affecting the results of our perceptual experiments.

Before starting the experiment, participants read and signed the informed consent form approved by the local ethics committee. Then, they were given information on how motion capture data is created and told that some of the animations had been modified so that in some of the animations, the velocity of the ball could appear excessively slow or fast with respect to preparatory motion. After each trial, participants indicated whether they thought that the trajectory of the ball was correct or incorrect regarding the preparatory motion by clicking the left or right button of the mouse, respectively. To facilitate the task, participants were allowed to feel the weight of a real tennis ball before and during the experiment, and they were told that the tennis ball displayed in the animation had the same weight as the real one. On average, each experimental session lasted about 25 minutes. 

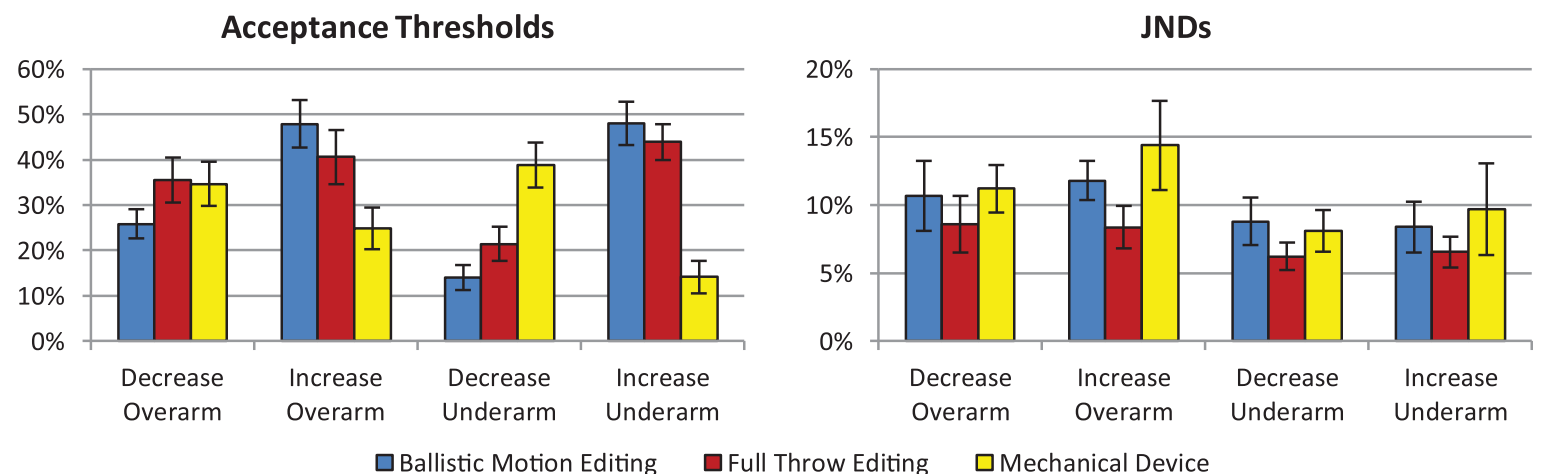

Fig. 2. Mean acceptance thresholds (left) and mean just noticeable differences (JNDs; right) for the four experimental conditions (horizontal axes) in experiments 1,2 , and 4 (vertical bars depict standard errors).

Table II. Mean Acceptance Thresholds (ATs) and Just Noticeable Differences (JNDs) with Standard Errors for Experiments 1, 2, and 4

\begin{tabular}{|c|l|r|r|r|r|r|c|}
\hline \multirow{2}{*}{} & \multicolumn{2}{|c|}{ Ballistic Motion Editing } & \multicolumn{2}{c|}{ Full Throw Motion Editing } & \multicolumn{2}{c|}{ Mechanical Device } \\
\cline { 2 - 8 } & Decrease & Increase & \multicolumn{1}{c|}{ Decrease } & \multicolumn{1}{c|}{ Increase } & \multicolumn{1}{c|}{ Decrease } & Increase \\
\hline \multirow{2}{*}{ Overarm } & ATs & $25.8 \pm 3.2 \%$ & $47.9 \pm 5.3 \%$ & $35.6 \pm 4.9 \%$ & $40.6 \pm 6.0 \%$ & $34.7 \pm 4.8 \%$ & $24.8 \pm 4.6 \%$ \\
\cline { 2 - 8 } & JNDs & $10.7 \pm 2.6 \%$ & $11.8 \pm 1.4 \%$ & $8.6 \pm 2.1 \%$ & $8.4 \pm 1.6 \%$ & $11.2 \pm 1.8 \%$ & $14.4 \pm 3.3 \%$ \\
\hline \multirow{2}{*}{ Underarm } & ATs & $14.0 \pm 2.7 \%$ & $48.0 \pm 4.8 \%$ & $21.4 \pm 3.8 \%$ & $43.9 \pm 4.0 \%$ & $38.8 \pm 5.0 \%$ & $14.2 \pm 3.6 \%$ \\
\cline { 2 - 7 } & JNDs & $8.8 \pm 1.7 \%$ & $8.4 \pm 1.9 \%$ & $6.2 \pm 1.0 \%$ & $6.6 \pm 1.1 \%$ & $8.1 \pm 1.6 \%$ & $9.7 \pm 3.4 \%$ \\
\hline
\end{tabular}

Fifteen volunteers took part in this experiment (eight women and seven men, aged between 21 and 28 years). They were all naïve to the purpose of the experiment, came from various educational backgrounds, and were compensated for their time with a five-euro cash payment or book token.

\subsection{Results}

For each experimental condition, we used the Matlab psignifit toolbox [Brainard 1997; Pelli 1997; Kleiner et al. 2007] to fit a logistic psychometric curve to the individual data of each participant, then computed individual acceptance thresholds and JNDs. The averaged results across participants for each experimental condition are shown in Figure 2 (blue bars) and reported in Table II. To evaluate how speed modification and throw influenced the acceptance threshold, we performed a two-way repeated measures analysis of variance (ANOVA) on individual estimated acceptance thresholds with withinsubjects factors: 2 Sign $\times 2$ Throw. We used Newman-Keuls post hoc tests to further explore interaction effects. Table III summarizes the most important statistically significant results.

We found main effects of Sign and Throw, as well as an interaction effect between them, which showed that participants are significantly more sensitive to decreases in the release velocity of the ball than to increases, especially for the underarm throw. A possible explanation of this result will be provided in Section 9.2. The same analysis was performed on JNDs, but no main or interaction effects were found, showing that the response strategy was consistent over the four experimental conditions.

The results suggest that observers are relatively tolerant to increases in the release velocity of the ball, independently of the kind of throw ( $\approx 48 \%$ increase in the original release velocity was tolerated $50 \%$ of the time). Tolerance for decreases is generally lower (with $-25.8 \%$ of the original release velocity accepted $50 \%$ of the time for the overarm throw), and particularly low for the underarm throw ( $-14 \%)$. 
Table III. Main Significant Results of the Presented Experiments

The full statistical analysis is available in supplementary materials.

\begin{tabular}{|c|c|c|c|}
\hline \multicolumn{4}{|c|}{ Experiment 1: Ballistic Motion Editing } \\
\hline & Effect & $F$-Test & Post hoc \\
\hline \multirow{3}{*}{ ATs } & Throw & $F_{(1,14)}=7.4314, p<0.05$ & Greater sensitivity to modifications in the underarm throw \\
\hline & Sign & $\begin{array}{l}F_{(1,14)}=41.834 \\
p<0.00001\end{array}$ & Greater sensitivity to decreases than to increases \\
\hline & Throw $\times$ Sign & $F_{(1,14)}=5.4720, p<0.05$ & $\begin{array}{l}\text { Greater sensitivity to decreases than to increases, especially for } \\
\text { the underarm throw }\end{array}$ \\
\hline \multicolumn{4}{|c|}{ Experiment 2: Full Throw Editing } \\
\hline & Effect & $F$-Test & Post hoc \\
\hline \multirow[b]{2}{*}{ ATs } & Throw & $F_{(1,14)}=5.1102, p<0.05$ & Greater sensitivity to modifications in the underarm throw \\
\hline & Throw $\times$ Sign & $\begin{array}{l}F_{(1,14)}=12.593 \\
p<0.005\end{array}$ & $\begin{array}{l}\text { Greater sensitivity to decreases in the underarm throw than in } \\
\text { the other experimental conditions }\end{array}$ \\
\hline \multicolumn{4}{|c|}{ Experiment 3: Angle of Release Editing } \\
\hline & Effect & $F$-Test & Post hoc \\
\hline ATs & Throw $\times$ Sign & $F_{(1,14)}=52.333, p \approx 0$ & $\begin{array}{l}\text { Greater sensitivity to angular decreases in the overarm throw, } \\
\text { but to increases in the underarm throw }\end{array}$ \\
\hline JNDs & Sign & $F_{(1,14)}=5.6450, p<0.05$ & $\begin{array}{l}\text { Sharper distinction between correct and incorrect animations for } \\
\text { angular increases than for angular decreases }\end{array}$ \\
\hline \multicolumn{4}{|c|}{ Experiment 4: Mechanical Device } \\
\hline & Effect & $F$-Test & Post hoc \\
\hline \multirow[t]{2}{*}{ ATs } & Sign & $F_{(1,14)}=6.6838, p<0.05$ & Greater sensitivity to increases than to decreases \\
\hline & Throw $\times$ Sign & $F_{(1,14)}=6.2372, p<0.05$ & $\begin{array}{l}\text { Greater sensitivity to increases than to decreases, especially for } \\
\text { the underarm throw }\end{array}$ \\
\hline \multicolumn{4}{|c|}{ Experiment 1 vs. Experiment 2 (between-subjects factor experimental group) } \\
\hline & Effect & $F$-Test & Post hoc \\
\hline \multirow{4}{*}{ ATs } & Throw & $\begin{array}{l}F_{(1,28)}=12.268 \\
p<0.005\end{array}$ & Greater sensitivity to modifications in underarm throws overall \\
\hline & Sign & $\begin{array}{l}F_{(1,28)}=26.010 \\
p<0.00005\end{array}$ & Greater sensitivity to decreases than to increases overall \\
\hline & Throw $\times$ Sign & $\begin{array}{l}F_{(1,28)}=17.203 \\
p<0.0005\end{array}$ & $\begin{array}{l}\text { Greater sensitivity to decreases than to increases overall, } \\
\text { especially for underarm throws }\end{array}$ \\
\hline & \multicolumn{3}{|c|}{ No main effect or interaction effect of variable Experimental Group } \\
\hline JNDs & Throw & $F_{(1,28)}=6.4311, p<0.05$ & $\begin{array}{l}\text { Sharper distinction between correct and incorrect animations in } \\
\text { underarm throws overall }\end{array}$ \\
\hline \multicolumn{4}{|c|}{ Experiment 1 vs. Experiment 4 (between-subjects factor experimental group) } \\
\hline & Effect & $F$-Test & Post hoc \\
\hline \multirow{3}{*}{ ATs } & Throw & $\begin{array}{l}F_{(1,28)}=9.7805 \\
p<0.005\end{array}$ & Greater sensitivity to modifications in underarm throws overall \\
\hline & Sign $\times$ E.G. & $F_{(1,28)}=32.390, p \approx 0$ & $\begin{array}{l}\text { Greater sensitivity to increases than to decreases for mechanical } \\
\text { throws, the opposite for human throws }\end{array}$ \\
\hline & $\mathrm{T} \times \mathrm{S} \times \mathrm{E} . \mathrm{G}$ & $\begin{array}{l}F_{(1,28)}=11.689 \\
p<0.005\end{array}$ & $\begin{array}{l}\text { Difference between human and mechanical throws more } \\
\text { prominent in underarm throws }\end{array}$ \\
\hline JNDs & Throw & $F_{(1,28)}=5.8367, p<0.05$ & $\begin{array}{l}\text { Sharper distinction between correct and incorrect animations in } \\
\text { underarm throws overall }\end{array}$ \\
\hline
\end{tabular}


Our findings show that observers' sensitivity to speed modifications depends on the fine features (overarm vs. underarm) of the action being performed (a throw).

To sum up, animators can modify the release velocity of the ball to achieve big increases in the throwing distance - that is, the horizontal distance between the thrower and the landing position of the ball. In our experiment, a $48 \%$ increase in the original release velocity resulted in an $85 \%$ increase in the overarm throwing distance and a $108 \%$ increase in the underarm throwing distance (more than twice the original distance). However, observers are more sensitive to slowed-down motions: a 25.8\% decrease in the original release velocity resulted in a $35 \%$ decrease in the overarm throwing distance, and a $14 \%$ decrease resulted in a $24 \%$ decrease in the underarm throwing distance. The latter result suggests that this simple editing operation can be used to decrease the throwing distance of underarm throws by a relatively small amount.

Recall that in the second experiment of our previous study [Vicovaro et al. 2012], we left the motion of the thrower unchanged and separately modified the horizontal and vertical components of the release velocity of the ball, thus also changing its angle of release. A comparison with the results of the current experiment reveals that observers are more tolerant to modifications when both horizontal and vertical components are modified by the same percentage. This suggests that modifying the absolute magnitude of release velocity of the ball, while leaving its angle of release unchanged, produces more perceptually plausible animations than modifying both physical parameters.

\section{EXPERIMENT 2: FULL THROW EDITING}

The Ballistic Motion Editing experiment created a mismatch between preparatory and ballistic motion, as the former remained unchanged while the latter was modified. In this second experiment, we wanted to evaluate the effect of simultaneously modifying both the preparatory and ballistic motions. We therefore edited the preparatory motion using DTW to modify the speed of the biological throwing motion and manipulated the release velocity of the ball accordingly. Although this alteration reduced or eliminated the mismatch between preparatory and ballistic motions, it may also have altered the naturalness of the resulting motion of the virtual human character and of the ball. Therefore, participants in this experiment were asked to indicate whether the presented animation was natural or modified. As concerns the difference between the forced choice tasks that we used in Experiments 1 and 2 (i.e., correct/incorrect vs. natural/modified), we reasoned that the former is more appropriate when referring to a specific relation between two variables, such as the consistency between the preparatory and the ballistic motion, whereas the latter is more appropriate when referring to the perceptual appearance of biological motion, such as in the present experiment. This experiment was also reported in Vicovaro et al. [2012] but is presented here with a larger number of participants. The results reveal that this new editing operation does not significantly increase the magnitude at which speed modifications are tolerated with respect to ballistic motion editing.

\subsection{Methods and Stimuli}

6.1.1 Dynamic Time Warping. In this experiment, we are interested in modifying simultaneously both preparatory and ballistic motions. As the release velocity of the projectile depends on the preparatory motion, we edited the preparatory motion using DTW to modify the speed of the biological throwing motion and manipulated the release velocity of the ball accordingly. DTW handles nonuniform compressions or dilatations of parts of a motion by varying the speed modification over time and is commonly used in computer animation to synchronize motion sequences with different durations [Bruderlin and Williams 1995]. In the case of throwing motions, the release velocity of the projectile depends on the preparatory motion of the human character. According to physics, this corresponds to modifying the horizontal and vertical components of release velocity by the same percentage as 
the speed modification of the preparatory motion. This modifies the magnitude of the release velocity without changing the angle of release of the projectile. As the release velocity is influenced only by the throwing gesture, we modified the speed of the motion of the human character only during the period of time bounded by two local minima of the velocity of the arm, which corresponded to the beginning and the end of the throwing gesture. This period included (approximately halfway) the moment of release. To manipulate the release velocity of the throw, we modified the duration of the throwing action by the corresponding amount and recomputed the new time of release. To ensure continuity with the other phases of the motion, we defined a time warping function using a monotonous and $C^{2}$ continuous spline. The modified parabolic trajectory of the projectile was then recomputed according to Equation 1 using the modified release velocities. Such editing is reasonably straightforward to perform and would therefore be typical in real-time applications such as games.

6.1.2 Stimuli. In this experiment, we used the same psychophysical method as in Experiment 1. Therefore, the experiment included eight staircases: 2 Staircase direction (ascending, descending) $\times 2$ Throw (overarm, underarm) $\times 2$ Sign (decrease, increase), with the Magnitude of modifications of the original motion speed varying between $0 \%$ and $90 \%$. The virtual human character, the camera viewpoint, the experimental apparatus, and the other details of the animations were also the same as in Experiment 1.

Before starting the experiment, participants read and signed the informed consent form. Then, they were given information on how motion capture data is created and were told that some of the animations had been modified in such a way that the throwing motion of the virtual character could appear excessively slow or fast with respect to a natural throwing motion of a tennis ball. After each trial, participants were to indicate whether the presented animation was natural or modified by clicking the left or right button of the mouse, respectively. As in Experiment 1, participants were allowed to feel the weight of a real tennis ball before and during the experiment. Fifteen volunteers took part in this experiment (seven women and eight men, aged between 21 and 50 years). They were all naïve to the purpose of the experiment, came from various educational backgrounds, and were compensated for their time with a five-euro cash payment or book token. None of them had participated in Experiment 1.

\subsection{Results}

As in Experiment 1, we fitted logistic psychometric curves to the individual data of each participant and computed individual acceptance thresholds and JNDs. The averaged results across participants for each experimental condition are shown in Figure 2 (red bars) and reported in Table II. We also performed the same statistical analyses as in Experiment 1, and Table III summarizes the main statistically significant results.

We found a main effect of Throw and an interaction effect between Throw and Sign, which showed that participants are more sensitive to decreases in the speed of the underarm throw than to the other experimental manipulations. The same analysis was performed on JNDs, and we found no main or interaction effects, demonstrating again that the response strategy was consistent over the four experimental conditions. As in Experiment 1, these findings suggest that observers' sensitivity to speed modifications depends on the specific features (i.e., overarm vs. underarm) of the action being performed.

In order to compare the results from Experiments 1 and 2 (Ballistic Motion Editing vs. Full Throw Editing), we performed a three-way mixed-effect ANOVA on individual estimated acceptance thresholds with within-subjects factors Sign and Throw and between participants factor Experimental Group. We used Newman-Keuls post hoc tests to further explore interaction effects, and Table III summarizes the main significant results. The comparison between blue and red bars in Figure 2 reveals the 
similarity between the results of Experiments 1 and 2. As throwing distance is a monotonically increasing function of release velocity, the same magnitude of manipulation produced the same modification of the throwing distance in the two experiments. The goal of this statistical comparison is to determine which of the two editing operations is the most suitable for modifying the original release velocity of the ball (and thus its throwing distance) while preserving the perceptual plausibility of throwing animations. The use of the same psychophysical method (i.e., randomly interleaved staircases) justifies the statistical comparison of individual acceptance thresholds.

We found no main or interaction effects of variable Experimental Group, which means that modifying the preparatory motion of the virtual thrower by the same percentage as the release velocity of the ball does not significantly increase the magnitude at which modifications are tolerated, with respect to modifying only the release velocity. Recall instead that in our previous study [Vicovaro et al. 2012], we found higher tolerance to modifications when both the preparatory motion and the release velocity of the ball were manipulated, compared to when only the former was manipulated. This shift in the results may partially be due to the diminished sensitivity to distortions in the Ballistic Motion Editing experiment, which resulted from modifying only the magnitude of release velocity rather than both magnitude and angle of release (see Section 5.2). Moreover, a possible explanation for the similarity in the results of the Ballistic Motion and the Full Throw Editing experiments is that in the latter, the gain in perceptual plausibility resulting from the elimination of the mismatch between preparatory and ballistic motion was compensated for by the loss in perceptual plausibility resulting from the manipulation of the biological throwing motion. However, a deeper explanatory hypothesis will be discussed in Section 9.2.

We also found a main effect of Throw and Sign, as well as a Sign $\times$ Throw interaction, similar to those already discussed previously and in Section 5.2. The same analysis was performed on JNDs and showed a main effect of Throw, meaning that, on average, participants' distinction between correct and incorrect animations is sharper for underarm than for overarm throws. However, the analysis showed no main or interaction effects of variable Experimental Group, meaning that the response strategy was consistent over the two experimental groups.

As editing the full throw does not significantly improve the plausibility of edited animations, while introducing a more complex correction, editing the ballistic motion only is therefore more advisable. The only exception seems to be when decreasing the speed of the overarm throw, for which full throw editing seems to be more effective than the ballistic motion editing, albeit not to a statistically significant extent (mean acceptance thresholds equal to $-25.8 \%$ and $-35.6 \%$, respectively).

\section{EXPERIMENT 3: ANGLE OF RELEASE EDITING}

Ballistic Motion Editing (Experiment 1) and Full Throw Editing (Experiment 2) have proven to be quite ineffective for producing plausibly shortened throwing animations, especially for underarm throws. To test the possibility of further decreasing the original throwing distance while preserving the perceptual plausibility of the animation, we explore manipulations of the angle of release of the ball while leaving the magnitude of the release velocity and the preparatory motion unchanged (Figure 3). Experiment 3 complements Experiment 1, as in the former we modify the angle of release of the ball while leaving the magnitude of release velocity unchanged, whereas in the latter we did the opposite. The results show that increasing the angle of release is effective for increasing the plausibility of shortened underarm throwing animations.

\subsection{Methods and Stimuli}

7.1.1 Angle Modifications. In this experiment, we leave the preparatory motion of the virtual character unchanged and manipulate only the angle of release of the throw. Such manipulations require 

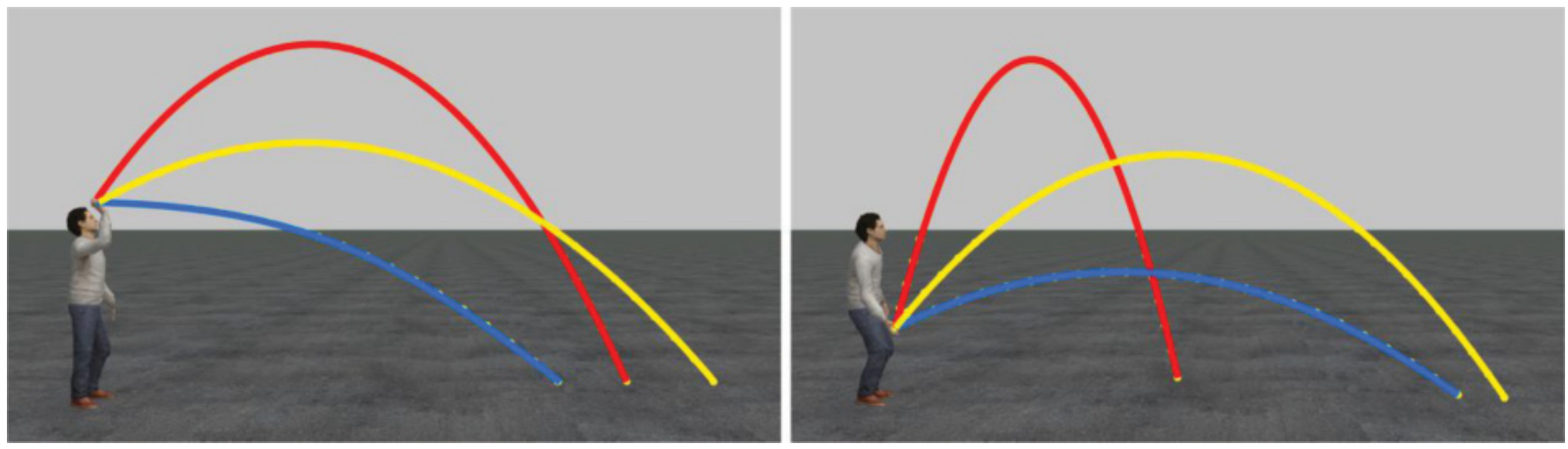

Fig. 3. Examples of angle of release manipulations for overarm (left) and underarm (right) throws. Yellow: original captured trajectory. Blue: A 25-degree decrease of the angle of release. Red: A 25-degree increase of the angle of release.

modifications of both the horizontal and vertical components of the release velocity. To keep the absolute release velocity $\|v\|$ unchanged, we consider the following equation:

$$
\|v\|=\sqrt{\left(v_{h}^{2}+v_{v}^{2}\right)},
$$

where $v_{h}$ and $v_{v}$ are the horizontal and vertical components of the release velocity, respectively. When changing the release angle by $\alpha$ degrees, the modified release velocities $v_{h}^{\prime}$ and $v_{v}^{\prime}$ are computed using the following equation:

$$
\left(\begin{array}{l}
v_{h}^{\prime} \\
v_{v}^{\prime}
\end{array}\right)=\left(\begin{array}{rr}
\cos (\alpha) & -\sin (\alpha) \\
\sin (\alpha) & \cos (\alpha)
\end{array}\right)\left(\begin{array}{l}
v_{h} \\
v_{v}
\end{array}\right)
$$

The modified parabolic trajectory of the ball is then recomputed according to Equation 1 using the modified release velocities. The captured overarm and underarm throws used in our experiments are characterized by an angle of release relative to the ground of 31.5 and 51.1 degrees, respectively, thereby producing the yellow parabolic trajectories depicted in Figure 3. Decreasing the angle of release produces shallower trajectories, whereas increasing the angle of release produces higher and shorter (bell-shape) trajectories (see Figure 3 and the supplementary video for detailed examples). Importantly, angular decreases and increases can both produce large decreases in the original throwing distance, whereas only a small range of angle modifications can produce increases in the throwing distance: the longest distances are obtained by an increase of 5 degrees of the angle of release for the overarm throw (distance increased by $0.9 \%$ ) and a decrease of 9.5 degrees for the underarm throw (distance increased by $4.7 \%$ ).

7.1.2 Stimuli. In this experiment, we used the same psychophysical method as in Experiment 1. Therefore, the experiment consisted of eight staircases: 2 Staircase direction (ascending, descending) $\times 2$ Throw (overarm, underarm) $\times 2$ Sign (decrease, increase). Based on a pilot study, the Angle modifications varied between 0 and 60 degrees with respect to the angle of release of the corresponding captured throw. The virtual human character, the camera viewpoint, the experimental apparatus, and the other details of the animations were also the same as in Experiment 1.

After they read and signed the informed consent form, participants were given information on how motion capture data is created and were told that some of the animations had been modified so that in some of the animations the trajectory of the ball could appear implausible-that is, too high or too shallow with respect to preparatory motion. After each trial, participants were to indicate whether the 
Acceptance Thresholds

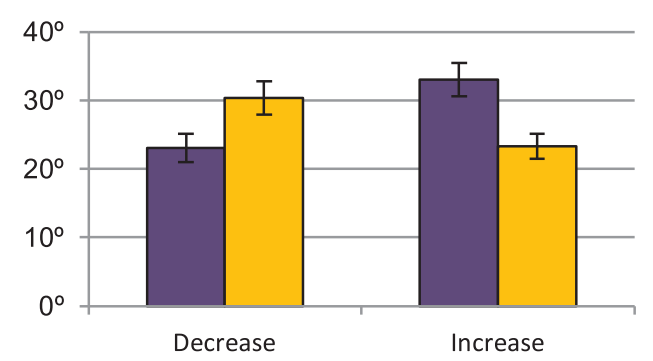

JNDs

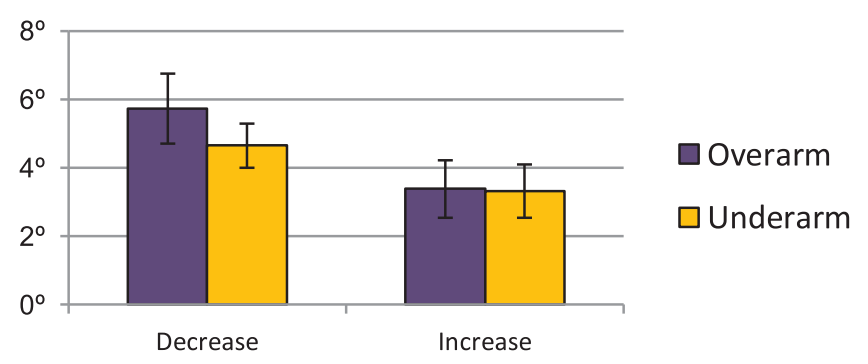

Fig. 4. Mean acceptance thresholds (left) and mean just noticeable differences (JNDs; right) for the Angle of Release Editing experiment (vertical bars depict standard errors).

trajectory of the ball was correct or incorrect with respect to the preparatory motion by clicking the left or right button of the mouse, respectively. To facilitate the task, participants were again allowed to feel the weight of a real tennis ball before and during the experiment, as before.

Fifteen volunteers took part in this experiment (seven women and 8 men, aged between 19 and 31 years). They were all naïve to the purpose of the experiment, came from various educational backgrounds, and were compensated for their time with a five-euro cash payment or book token. None of them had participated in Experiment 1 or 2.

\subsection{Results}

As in Experiment 1, we fitted logistic psychometric curves to the individual data of each participant and computed individual acceptance thresholds and JNDs. Figure 4 shows the mean acceptance thresholds and JNDs (across participants) for each experimental condition. We performed the same statistical analyses as in Experiment 1, and Table III summarizes the main statistically significant results.

We found no significant main effect, but there were significant interaction effects between Throw and Sign, which indicates that participants are more sensitive to decreases in the angle of release for the overarm throw, whereas they are more sensitive to increases in the angle of release for the underarm throw. A possible explanation of this result will be provided in Section 9.2. The same analysis was performed on JNDs and showed a main effect of variable Sign, meaning that, on average, participants' distinction between correct and incorrect animations is sharper for increases in the angle of release than for decreases. However, the most important result of this experiment is the increase, in absolute value, of the largest accepted reduction in the throwing distance for the underarm throw. Indeed, a 23.3 degree increase in the angle of release (acceptance threshold) results in a $48.5 \%$ decrease in the original throwing distance, which is higher in absolute value than the $24 \%$ distance decrease we found in Experiment 1 (see Section 5.2). To test for statistical differences, we first transformed individual acceptance thresholds from Experiments 1 and 3 into the corresponding modified distances. Then, we performed independent-sample $t$-tests on the modified distances by comparing decreases in the release velocity of the ball (Experiment 1) with decreases or increases in its angle of release (Experiment 3). The results of the $t$-tests confirmed that increases in the angle of release for the underarm throw do produce statistically shorter throws for the $50 \%$ acceptance threshold $\left(t_{(28)}=4.131, p<0.0005\right)$, but they also showed that decreasing the release velocity of the ball is more efficient than decreasing its angle of release for shortening the overarm throw $\left(t_{(28)}=-2.716, p<0.05\right)$. 
Therefore, these findings suggest that increasing the angle of release is more efficient in the case of shortening the underarm throw, but modifying the angle of release does not prove as efficient as decreasing the magnitude of the release velocity for the overarm throw. In addition, this method does not help to increase the throwing distance of either overarm or underarm throws, as mentioned previously in Section 7.1.1. Importantly, modifications of the angle of release can be used not only for shortening underarm throws but also for obtaining shallower or higher (bell-shape) parabolic trajectories for both kinds of throws (see the supplementary video for detailed examples). In the present study, we do not combine modifications of the magnitude of release velocity and of the angle of release, because this has already proven to be less effective than modifying the magnitude of release velocity only (see Section 5.2).

\section{EXPERIMENT 4: MECHANICAL DEVICE}

Experiments 1 and 2 showed that observers' sensitivity to distortions depends on the interaction between the type of throw and the sign of speed modification, with participants being highly sensitive to speed decreases in the underarm throw. To explore if such sensitivity is specific to human motion, we now consider observers' sensitivity to modifications of the release velocity of the ball when the preparatory motion involves a mechanical device instead of a human character. The participants are presented with a tennis ball descending along a virtual wooden ramp (preparatory motion) at the end of which it is released in free fall (ballistic motion). As in Experiment 1, we modify the release velocity of the ball while leaving its angle of release and the preparatory motion unchanged. The purpose of this experiment is similar to that of Reitsma et al. [2008], who found that observers are more sensitive to manipulations of physical parameters in biological ballistic motion (a jumping virtual human character) than in the case of simple mechanical ballistic motion (a ball released from a cannon or from a ramp). Our focus is more on the sensitivity to mismatches between preparatory and ballistic motions rather than on the ballistic motion itself. The results reveal a different pattern of sensitivity to speed modifications with respect to the human throw, with participants being more sensitive to speed increases than to speed decreases. However, the average level of sensitivity to modifications was not significantly different with respect to the human thrower.

\subsection{Methods and Stimuli}

8.1.1 Ramps Design. In this experiment, participants are presented with a tennis ball descending along a virtual wooden ramp (preparatory motion). At the end of the ramp, the ball is then released in free fall (ballistic motion) with a velocity matching one of those from the previous experiments. From several possible throwing mechanical devices (e.g., cannon, catapult, crossbow), we chose a ramp because it allows the observer to perceive much of the preparatory motion. To compare the results of this experiment with those of Experiment 1, the ball is released with physical parameters (position, velocity, and angle) matching those of the throwing animations in Experiment 1 . We therefore created two ramp models to match the overarm and underarm throws of Experiment 1 (see Table I and Figure 1). For the sake of simplicity, the two ramp conditions will be called overarm and underarm, respectively, analogous to the corresponding human throws. Parameters for the design of the ramps are presented in Figure 5. The starting position of the ball and its descending motion are computed from the physical simulation of a free fall of the ball on the surface of the ramp under the effect of gravity (no friction) to match the velocity at the time of release. As in Experiment 1, the ballistic motion was modified by manipulating the original release velocity of the ball while the preparatory motion remained unchanged. Both the horizontal and the vertical components of the original release velocity were modified by the same percentage, thus leaving the angle of release unchanged. The modified parabolic trajectory of the projectile was then recomputed according to Equation 1 using the modified release velocities. 


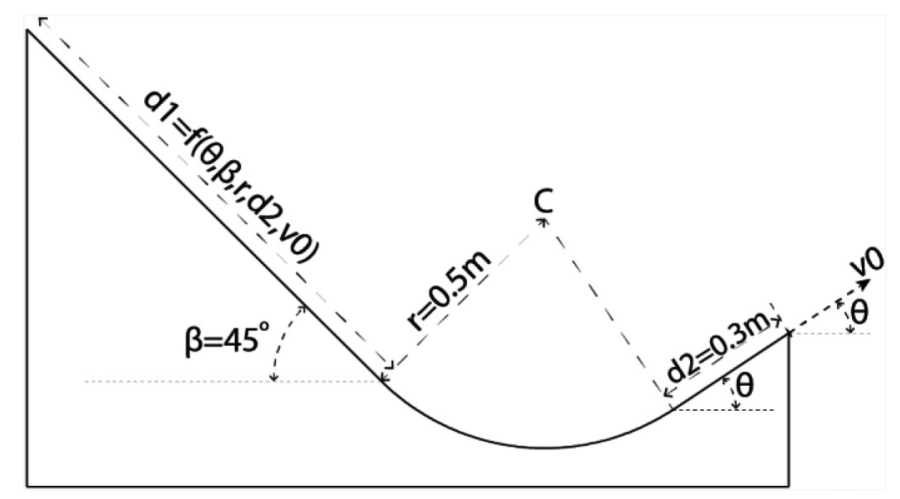

Fig. 5. Parameters for designing the virtual ramps: $\beta, r$, and $d 2$ were the same for all the ramps, whereas $d 1$ was computed to ensure that a ball starting at the top of ramp and falling on its surface under the effect of gravity (no friction) would be released with a velocity $v 0$ (angle of release $\theta$ ).

8.1.2 Methods and Participants. We used the same psychophysical method as in Experiment 1. Therefore, the experiment consisted of eight staircases: 2 Staircase direction (ascending, descending) $\times$

2 Ramp throw (overarm, underarm) $\times 2$ Sign (decrease, increase). The Magnitude of modifications of the original motion speed varied between $0 \%$ and $90 \%$. The camera viewpoint, the experimental apparatus, and the other details of the animations were also the same as in Experiment 1.

After they read and signed the informed consent form, participants were told that they would be presented with animations simulating a tennis ball descending along a wooden ramp, and that in some of the animations the velocity of the ball after the release could appear excessively slow or fast regarding the velocity of the descent. After each trial, participants indicated whether the trajectory of the ball was correct or incorrect with respect to the descent by clicking the left or right button of the mouse, respectively. To facilitate the task, participants were again allowed to feel the weight of a real tennis ball before and during the experiment, as before.

Fifteen volunteers took part in this experiment (eight women and seven men, aged between 20 and 30 years). They were all naïve to the purpose of the experiment, came from various educational backgrounds, and were compensated for their time with a five-euro cash payment or book token. None of them had participated in the previous experiments.

\subsection{Results}

As in Experiment 1, we fitted logistic psychometric curves to the individual data of each participant and computed individual acceptance thresholds and JNDs. The averaged results across participants for each experimental condition are shown in Figure 2 (yellow bars) and reported in Table II. To evaluate how speed modification and throw type influence the acceptance threshold, we performed a two-way repeated measures ANOVA on individual estimated acceptance thresholds with within-subjects factors: 2 Sign $\times 2$ Throw. We used Newman-Keuls post hoc tests to further explore interaction effects. Table III summarizes the main statistically significant results.

We found a main effect of Sign and an interaction effect between Throw and Sign, which showed that participants are significantly more sensitive to increases in the release velocity of the ball than to decreases, especially for the underarm throw. The same analysis was performed on JNDs and showed no main or interaction effects, revealing that the response strategy was consistent over the four experimental conditions. 
The results suggest that observers are relatively tolerant to decreases in the release velocity of the ball, independently of the kind of throw (acceptance thresholds equal to $-34.7 \%$ and $-38.8 \%$ for overarm and underarm throws, respectively). Tolerance for increases is generally lower (acceptance threshold for the underarm throw equal to $24.8 \%$ ), and particularly low for the underarm throw (14.2\%). Again, our findings show that observers' sensitivity to speed modifications depends on the specific features (overarm vs. underarm) of the physical event being presented.

To compare the results of human and mechanical throws, we performed a three-way mixed-effect ANOVA on individual estimated acceptance thresholds with within-subjects factors Sign and Throw, and between participants factor Experimental Group (Experiment 1-human throw or Experiment 4mechanical throw). We used Newman-Keuls post hoc tests to further explore interaction effects, and Table III summarizes the main significant results. The comparison between blue and yellow bars in Figure 2 reveals the difference between the results of Experiments 1 and 4 .

We found no main effect of variable Experimental Group, which means that when averaged across all experimental conditions, participants' sensitivity to mismatches between preparatory and ballistic motions does not depend on the "biological" or "mechanical" nature of the thrower. This result is somewhat at odds with Reitsma et al. [2008], who found that observers' sensitivity to errors in ballistic motion is greater for biological ballistic motion than for simple physical ballistic motion. We refer the reader to Section 9.3 for a more detailed discussion of this discrepancy.

We also found a main effect of Throw, as well as a Sign $\times$ Experimental Group interaction and a three-factor interaction, where post hoc analysis showed that participants are more sensitive to decreases than to increases in the release velocity of biological throws, whereas the opposite occurs in mechanical throws, especially for underarm throws. In other words, the pattern of sensitivity to mismatches in mechanical throws is the opposite of that characterizing biological throws (see Figure 2). This finding is particularly interesting and thus we refer the reader to Section 9.3 for a complete discussion. The same analysis was performed on JNDs and showed a main effect of Throw, meaning that, on average, participants' distinction between correct and incorrect animations is sharper for underarm than for overarm throws. However, the analysis showed no main or interaction effect of variable Experimental Group, meaning that the response strategy was consistent over the two experimental groups.

\section{DISCUSSION AND CONCLUSION}

Our study can provide useful guidelines for developers of games and VR applications by specifying thresholds for the perceptual plausibility of throwing animations. The results of Experiment 1 show that modifying the release velocity of the ball while leaving the preparatory motion unchanged can be used to achieve big increases in the throwing distance (e.g., a 108\% increase in the throwing distance for the underarm throw was considered to be correct $50 \%$ of the time). However, the throwing distance of underarm throws can be shortened only by a small amount $(-24 \%)$ using this simple kind of editing operation. As shown by the comparison between Experiments 1 and 2, modifying the preparatory motion with DTW in addition to modifying the release velocity of the ball does not improve the perceptual plausibility of such animations. The results of Experiment 3 show instead that it is possible to further shorten the throwing distance of underarm throws $(-48.5 \%)$ by increasing the angle of release of the ball while keeping the magnitude of release velocity and the preparatory motion unchanged. The results of Experiment 4 also provide guidelines for modifications of throwing animations with a mechanical device and suggest that in this case observers are more tolerant to speed decreases than to speed increases. In addition to these useful guidelines for developers of games and VR applications, our study also provides several interesting insights for researchers in visual perception of biological motion, which we will discuss in the following sessions. 


\subsection{Kinematic Cues and Response Accuracy}

In Experiment 1, we found that observers are relatively tolerant to increases in the release velocity of the ball, which lengthen the original throwing distance by a large amount. One may therefore infer that observers could not accurately predict the throwing distance of light objects from captured biological motion. In contrast, Runeson and Frykholm [1983] and Munzert et al. [2010] found that observers can accurately predict the throwing distance when presented with point-light characters throwing unseen $2.5 \mathrm{~kg}$ and $600 \mathrm{~g}$ projectiles. As shown by Munzert et al. [2010], observers can predict throwing distances by relying on kinematic cues of the preparatory motion of the thrower, which in fact are good predictors of this variable. These cues depend on the mass of the projectile, with the overall motion of the thrower's arm being a good predictor of the throwing distance in the case of a $600 \mathrm{~g}$ projectile [Munzert et al. 2010]. However, in the case of a tennis ball weighing approximately $60 \mathrm{~g}$, the fine motions of the thrower's wrist and fingers may also be important cues for this variable, because they significantly contribute to the overall force exerted by the thrower on the projectile, which is not the case with relatively heavy projectiles. This implies that the kinematic cues provided by the thrower's arm are less informative when the projectile is as light as a tennis ball, and that the kinematic cues provided by the thrower's wrist and fingers might be more relevant when predicting the throwing distance.

Munzert et al. [2010] showed that when informative kinematic cues are clearly visible, as is the case with the overall motion of the thrower's arm, observers' predictions of the throwing distance are fairly accurate. However, when the kinematic cues are more difficult to perceive, as it is for the fine motions of the thrower's wrist and fingers, it seems reasonable to expect less accurate judgments. Indeed, despite the realism of the virtual human character and the favourable camera viewpoint in Experiment 1 (Figure 1), the fine motions of the thrower's wrist and fingers were barely perceivable in our stimuli. This lack of perceptual information may explain why the judgments of participants in our study are less accurate compared with those found in previous studies. This seems to be the price to pay when building large-scale virtual scenarios involving full-body virtual human characters, thereby unavoidably decreasing the visibility of anatomical details. On the positive side, this loss of perceptual information allows animators to use motion editing operations without altering the realism of full-body throwing animations.

In our experiments, we only used a relatively light projectile and one camera viewpoint. Therefore, the hypothesis of a positive correlation between the amount of perceptual information provided by the preparatory motion of the thrower and participants' response accuracy needs to be tested in future studies, which will explore how projectiles of various masses and camera viewpoint affect the latter variable.

\subsection{Perceiving Virtual Throwing Animations: Perceptual Processes or Heuristic Strategies?}

According to Proffitt and Gilden [1989], when people are required to judge the realism of complex dynamic events, they tend to rely on heuristic strategies rather than on pure perceptual judgments. Heuristics are conscious or unconscious simplified strategies that allow people to respond while saving perceptual and/or cognitive resources. Heuristic strategies may be used when stimulus information is poor or ambiguous, and usually lead to less accurate responses compared to pure perceptual judgments. For instance, the lack of important kinematic cues, such as the motion of the thrower's wrist and fingers, might have led the participants in our experiments to base their judgments on memory of similar throwing actions they performed in the past (a heuristic strategy) rather than on pure perceptual information.

According to Runeson and Frykholm [1983], when observers are required to predict the throwing distance of a relatively heavy unseen projectile thrown by a point-light character, they rely on the 
kinematic cues provided by the preparatory motion of the character to make accurate predictions. In this case, observers rely on pure perceptual information rather than on heuristic strategies. However, our study differs from Runeson and Frykholm [1983] and Munzert et al. [2010] because of the use of a light projectile, which, as hypothesized in Section 9.1, should imply the loss of prominent kinematic cues. Because of the lack of important perceptual information, the participants of the first three experiments in our study (those involving a virtual human character) might have relied on heuristic strategies, such as referring to their past experience with throwing actions, rather than (or in addition to) pure perceptual processes.

Support for this hypothesis comes from the comparison between the results of Experiments 1 and 2 . In each of the four experimental conditions ( 2 Throw $\times 2$ Sign), the acceptance thresholds were very similar across the two experiments-that is, the threshold seemed to be independent of the kind of motion editing operation we used (ballistic motion only vs. full throw). This result is quite unexpected, as we asked participants two different questions-evaluating the correctness of the trajectory of the ball with respect to the preparatory motion in Experiment 1, and evaluating the naturalness of the animation in Experiment 2. It is possible that participants relied on pure perceptual information in both experiments, and that their sensitivity to these two distinct editing operations was the same in the four experimental conditions by mere coincidence. However, a more plausible hypothesis is that they used the same heuristic strategy (partially overlooking the specificity of the tasks) in both cases.

Our hypothesis is that in Experiment 1, participants' judgments were not based only on the perceived mismatch between preparatory and ballistic motion, just as in Experiment 2 they were not based only on the perceived naturalness of the throwing motion of the virtual character. Instead, in both experiments, participants' judgments might have been prominently influenced by the throwing distance. Thus, participants responded correct or natural when the ball fell within a certain range of throwing distances, and incorrect or modified when it fell outside this range. In Experiments 1 and 2, throwing distance is a monotonically increasing function of release velocity; hence, the same magnitude of manipulation produced the same modification of the throwing distance in the two experiments. The use of the same heuristic strategy that relied on throwing distance can thus explain the similarity between the acceptance thresholds.

If this hypothesis is correct, then the results of Experiments 1 and 2 suggest that each kind of throw is characterized by a specific range of acceptable throwing distances: for instance, the range of acceptable throwing distances for the underarm throw used in our experiments is between $-24 \%$ and $+108 \%$ of the original throwing distance. As shown by the results of Experiment 3, shorter throwing distances for underarm throws are accepted with larger angles of release. The latter result suggests that heuristic strategies do also take into account the angle of release: for each combination of kind of throw and magnitude of release velocity, there is a specific range of acceptable angles of release, which may explain the results of Experiment 3.

Heuristic strategies are likely to depend on participants' past experience with throwing actions. The relatively low tolerance for short throwing distances in underarm throws with a 51.1-degree angle of release (captured underarm throw) might depend on the difficulty that participants may have experienced when they tried to throw a tennis ball in this way. Short throws can be better carried out with overarm motions, or with underarm motions with larger angles of release. Our hypothesis is somewhat similar to that proposed by Knoblich and Flach [2001] and Munzert et al. [2010], who stated that visual perception of throwing actions depends on internal motor representations of the same actions, which are built from past motor experience.

According to our hypothesis, an increase in the amount of perceptual information provided by the preparatory motion of the thrower should prompt participants to rely on pure perceptual processes 
rather than on heuristic strategies. This can be tested in future studies by using heavier projectiles and camera viewpoints closer to the virtual thrower, which, for the reasons discussed in Section 9.2, should increase the amount of perceptual information available to participants. In that case, we would expect to obtain different acceptance thresholds in Experiments 1 and 2 as a consequence of the fact that the two experiments involve different perceptual processes.

\subsection{Biological and Mechanical Throws}

Heuristic strategies that are valid for human throws might not extend to mechanical throws. Indeed, when the virtual human character is replaced by a virtual ramp (Experiment 4), the participants are more sensitive to speed increases than to speed decreases, the opposite of what we found with the human character. Conversely, the results of Experiment 4 are in reasonable agreement with those reported by Reitsma et al. [2008], who investigated visual perception of distortions in ballistic motion. They found greater tolerance for additional deceleration than for additional acceleration, and greater tolerance for increases in the coefficient of gravity than for decreases, which may suggest the existence of a gravity overestimation bias. Note that in ballistic motion, only the vertical component of velocity is affected by the coefficient of gravity (Equation 1). In our study, the vertical component of velocity was greater for underarm than for overarm throws (Table I). If the hypothesis of a gravity overestimation bias is correct, then participants' greater tolerance for speed decreases over speed increases should be enhanced in underarm throws, which is exactly what we found in Experiment 4 (Figure 2 and Table III). However, the gravity overestimation bias seems to be valid only for mechanical throws; indeed, for human throws, we found the opposite pattern of results. This might simply indicate that the heuristic strategies for human throws are so pervasive that they overshadow the gravity overestimation bias.

An important outcome of the comparison between Experiments 1 and 4 is the similarity, in absolute value, between participants' sensitivity to speed manipulations for human and mechanical throws. This result is somewhat unexpected in light of the results reported by Reitsma et al. [2008], who showed that observers are more sensitive to manipulations of the coefficient of gravity when presented with human ballistic motion than when presented with simple physical ballistic motion. They interpreted these results as suggesting that people have greater experience with human motion than with simple physical motion, and that biological motion provides more perceptual information than simple physical motion. These are both plausible hypotheses, but our results also suggest that when fine stimulus information regarding biological motion lacks, as it was the case for wrist and fingers in our human throw experiments, observers' average sensitivity to physical distortions may be the same in human and simple physical motions.

\subsection{Future Work}

In our experiments, all of the captured biological animations were characterized by a throwing distance of $5 \mathrm{~m}$. To test the effect of manipulations on a wider range of throwing distances would have required an impractically large number of stimuli. For the same reason, we tested only the two most common throwing motions (overarm and underarm) from the vast set of possible throwing actions. The camera viewpoint was fixed and set to maximize the visual information available to the participants; tolerance to modified animations might be different with other arbitrary camera viewpoints. The mass of the ball was always the same and set to maximize the generalizability of the results to most VR applications. However, in light of the strong influence that this variable exerts on the preparatory motion, our results are probably specific to throwing animations involving light projectiles.

Although the previously mentioned choices were well justified for a first-stage experiment, future research on the perception of throwing animations will involve a wider range of throwing distances 
and actions, will evaluate the effect of the camera viewpoint on the perception of physical distortions, and will evaluate the effect of the mass of the projectile on acceptance thresholds.

\section{ACKNOWLEDGMENTS}

We wish to thank all reviewers for their comments and the participants in our experiments. This work was sponsored by Science Foundation Ireland as part of the Captavatar project (S.F.I. 10/IN.1/13003).

\section{REFERENCES}

R. Barzel, J. F. Hughes, and D. N. Wood. 1996. Plausible motion simulation for computer graphics animation. In Proceedings of the Eurographics Workshop on Computer Animation and Simulation. 183-197.

Bozzi, P. 1959. Analisi fenomenologica del moto pendolare armonico. Rivista di Psicologia 52, 4, 281-302.

D. H. Brainard. 1997. The Psychophysics Toolbox. Spatial Vision 10, 433-436.

A. Bruderlin and L. Williams. 1995. Motion signal processing. In Proceedings of the 22nd Annual Conference on Computer Graphics and Interactive Techniques (SIGGRAPH'95). 97-104.

T. Chaminade, J. Hodgins, and M. Kawato. 2007. Anthropomorphism influences perception of computer-animated characters' actions. Social Cognitive and Affective Neuroscience 2, 3, 206-216.

J. Clement. 1982. Students' preconceptions in introductory mechanics. American Journal of Physics 50, 1, 66-71.

T. N. Cornsweet. 1962. The staircase method in psychophysics. American Journal of Psychology 75, 3, 485-491.

M. A. García-Pérez. 2001. Yes-no staircases with fixed step sizes: Psychometric properties and optimal setup. Optometry and Vision Science 78, 1, 56-64.

M. Gleicher. 1997. Motion editing with spacetime constraints. In Proceedings of the 1997 Aymposium on Interactive 3D Graphics (I3D'97). 139-.

M. Gleicher. 1998. Retargetting motion to new characters. In Proceedings of the 25th Annual Conference on Computer Graphics and Interactive Techniques (SIGGRAPH'98). 33-42.

M. Gleicher. 2001. Motion path editing. In Proceedings of the 2001 Aymposium on Interactive 3D Graphics (I3D'01). 195-202.

S. Guo and J. Robergé, 1996. A high-level control mechanism for human locomotion based on parametric frame space interpolation. In Proceedings of the Eurographics Workshop on Computer Animation and Simulation. 95-107.

H. Hecht and M. Bertamini. 2000. Understanding projectile acceleration. Journal of Experimental Psychology: Human Perception and Performance 26, 2, 730-746.

J. Hodgins, J. O'Brien, and J. Tumblin, 1998. Perception of human motion with different geometric models. IEEE Transactions on Visualization and Computer Graphics 4, 4, 307-316.

L. Hoyet, R. McDonnell, and C. O’Sullivan. 2012. Push it real: Perceiving causality in virtual interactions. ACM Transactions on Graphics 31, 4, Article 90.

L. Hoyet, K. Ryall, R. McDonnell, and C. O'Sullivan. 2012. Sleight of hand: Perception of finger motion from reduced marker sets. In Proceedings of the ACM SIGGRAPH Symposium on Interactive 3D Graphics and Games (I3D'12). 79-86.

E. Hsu, M. da Silva, and J. Popovíc. 2007. Guided time warping for motion editing. In Proceedings of the 2007 ACM SIGGRAPH / Eurographics Symposium on Computer Animation (SCA'07). 45-52.

M. K. Kaiser, D. R. Proffitt, S. Whelan, and H. Hecht. 1992. Influence of animation on dynamical judgments. Journal of Experimental Psychology: Human Perception and Performance 18, 3, 669-689.

M. Kleiner, D. Brainard, and D. Pelli. 2007. What's new in Psychtoolbox-3? Perception 36, ECVP Abstract Supplement.

G. Knoblich and R. Flach. 2001. Predicting the effects of actions: Interactions of perception and action. Psychological Science 12, $6,467-472$.

T. Komura, E. Ho, and R. Lau. 2005. Animating reactive motion using momentum-based inverse kinematics: Motion capture and retrieval. Computer Animation and Virtual Worlds 16, 3-4, 213-223.

C. K. Liu, A. Hertzmann, and Z. Popovi. 2006. Composition of complex optimal multi-character motions. In Proceedings of the 2006 ACM SIGGRAPH / Eurographics Symposium on Computer Animation (SCA'06). 215-222.

A. Majkowska and P. Faloutsos. 2007. Flipping with physics: Motion editing for acrobatics. In Proceedings of the 2007 ACM SIGGRAPH / Eurographics Symposium on Computer Animation (SCA'07). 35-44.

M. McCloskey, A. Caramazza, and B. Green. 1980. Curvilinear motion in the absence of external forces: Naïve beliefs about the motion of objects. Science $210,4474,1139-1141$.

J. Munzert, T. Hohmann, and E. Hossner. 2010. Discriminating throwing distances from point-light displays with masked ball flight. European Journal of Cognitive Psychology 22, 2, 247-264. 
M. Nusseck, J. Lagarde, B. Bardy, R. Fleming, and H. H. Bülthoff. 2007. Perception and prediction of simple object interactions. In Proceedings of the Symposium on Applied Perception in Graphics and Visualization (APGV'07). 27-34.

C. O'Sullivan, J. Dingliana, T. Giang, and M. K. Kaiser. 2003. Evaluating the visual fidelity of physically based animations. ACM Transactions on Graphics 22, 3, 527-536.

S. Park, T.-H. Kim, H. Shin, and S. Shin. 2004. On-line motion blending for real-time locomotion generation. Computer Animation and Virtual Worlds 15, 3-4, 125-138.

D. G. Pelli. 1997. The VideoToolbox software for visual psychophysics: Transforming numbers into movies. Spatial Vision 10, 437-442.

N. Pollard and P. Reitsma. 2001. Animation of humanlike characters: Dynamic motion filtering with a physically plausible contact model. In Proceedings of the Yale Workshop on Adaptive and Learning Systems.

D. R. Proffitt and D. L. Gilden. 1989. Understanding natural dynamics. Journal of Experimental Psychology: Human Perception and Performance 15, 384-393.

P. S. A. Reitsma, J. Andrews, and N. S. Pollard. 2008. Effect of character animacy and preparatory motion on perceptual magnitude of errors in ballistic motion. Computer Graphics Forum 27, 2, 201-210.

P. S. A. Reitsma and C. O'Sullivan. 2009. Effect of scenario on perceptual sensitivity to errors in animation. ACM Transactions on Applied Perception 6, 3, 15:1-15:16.

S. Runeson and G. Frykholm. 1983. Kinematic specification of dynamics as an informational basis for person-and-action perception: Expectation, gender recognition, and deceptive intention. Journal of Experimental Psychology: General 112, 4, 585-615.

H. J. Shin and H. S. Oh. 2006. Fat graphs: Constructing an interactive character with continuous controls. In Proceedings of the 2006 ACM SIGGRAPH / Eurographics Symposium on Computer Animation (SCA'06). 291-298.

H. J. Shin, L. Kovar, and M. Gleicher. 2003. Physical touch-up of human motions. In Proceedings of the 11th Pacific Conference on Computer Graphics and Applications (PG'03). 194-.

S. Tak and H-S. Ko. 2005. A physically-based motion retargeting filter. ACM Transactions on Graphics 24, 1, 98-117.

M. Vicovaro, L. Hoyet, L. Burigana, and C. O'Sullivan. 2012. Evaluating the plausibility of edited throwing animations. In Proceedings of the ACM SIGGRAPH / Eurographics Symposium on Computer Animation (SCA'12). 175-182.

K. Yamane and Y. Nakamura. 2003. Dynamics filter-concept and implementation of online motion generator for human figures. IEEE Transactions on Robotics and Automation 19, 3, 421-432.

T. Y. Yeh, G. Reinman, S. J. Patel, and P. Faloutsos. 2009. Fool me twice: Exploring and exploiting error tolerance in physics-based animation. ACM Transactions on Graphics 29, 1, 5:1-5:11.

V. Zordan, A. Majkowska, B. Chiu, and M. Fast. 2005. Dynamic response for motion capture animation. ACM Transactions on Graphics 24, 3, 697-701.

Received November 2013; revised April 2014; accepted April 2014 